\title{
A Review of Cardiac and Non-Cardiac Causes of Troponin Elevation and Clinical Relevance Part II: Non Cardiac Causes
}

\begin{abstract}
Non-cardiac causes of elevated troponins are often overlooked, yet elevated troponins have been shown to closely correlate with the prognosis of these conditions. The clinical significance of elevated troponins in non-cardiac conditions are not completely known. Also, it is still unclear if these clinical conditions need to be managed differently when they lead to an increase in troponin levels. Some of the non-cardiac conditions in which elevated troponins are commonly found include: pulmonary embolism, end stage renal disease, Sepsis/ Systemic inflammatory response syndrome (SIRS) and critically ill patient, rhabdomyolysis, physical exercise, burns, drug toxicity and stroke. This article discusses the suggested etiology of elevated cardiac troponins in these conditions and the prognostic values they carry.
\end{abstract}

Keywords: Pulmonary embolism; End stage renal disease; Sepsis; Critically ill patient; Rhabdomyolysis; Physical exercise; Burns; Drug toxicity and stroke

Review Article
Volume 11 Issue 1 - 2018

Joyce Akwe ${ }^{1 *}$, Brittne Hallford ${ }^{2}$, Erin Kim ${ }^{1}$
and Amy Miller ${ }^{1}$
${ }^{1}$ Atlanta Veterans Affairs Medical Center, Emory University
School of Medicine, Department of Medicine, USA
'Emory University School of Medicine, Department of
Medicine, USA
*Corresponding author: Joyce Akwe, Atlanta Veterans
Affairs Medical Center, Emory University School of Medicine,
Department of Medicine, Atlanta GA, USA, Email:
Joyce.akwe@emory.edu
Received: December 19, 2017 | Published: January 08,
2018

\section{Introduction}

Not every troponin elevation is due to acute myocardial infarction (MI). Several other clinical cardiac conditions such as arrhythmias, cardiac ablation, cardiac contusion, infiltrative cardiac disorders, defibrillation shocks and many other conditions as listed in our previous article can lead to increase troponins. Elevated cardiac troponins have also been commonly found in non-cardiac conditions such as pulmonary embolism, end stage renal disease, Sepsis/ Systemic inflammatory response syndrome (SIRS) and critically ill patient, rhabdomyolysis, physical exercise, burns, drug toxicity and stroke. Both cTnI and cTnT have very high diagnostic accuracy for NSTEMI at presentation even though cTnI seemed to be superior in early presenters (<3hours), while cTnT seemed to be superior in late presenters [1]. In noncardiac conditions, troponins do not have a diagnostic accuracy. Pathophysiology of elevated cardiac troponins are not completely clear. One other common theme is the prognostic significance of elevated troponins in these patients. In these clinical conditions, elevated troponins have been found to be negative prognostic markers both for disease severity and complications. The only exception noted so far is exercise induced elevated troponins where we did not find any studies showing correlation between troponin elevation and any outcomes. This article discusses the significance of troponin elevation due to non-cardiac etiologies. These etiologies are needed for the differential diagnosis of elevated cardiac enzymes.

\section{Acute Pulmonary Embolism}

Elevated troponins are found in $30-50 \%$ of the patients diagnosed with pulmonary Embolism (PE). This large range in the incidence of increased troponins can be explained in the large range in the cut off value for troponins. In fact with the high sensitivity troponin (hs cTn) test, up to $64 \%$ in patients with PE had elevated cardiac enzymes (hs cTn $\geq 14 \mathrm{pg} / \mathrm{mL}$ ) [2,3]. Mareike et al. [2] showed that baseline hs cTn was higher in patients with an adverse 30-day outcome(death, need for catecholamines, endotracheal intubation, or cardiopulmonary resuscitation) compared with an uncomplicated course [2]. The mechanism for troponin increase in acute PE is still unclear. Some suggested mechanisms for troponin rise include pulmonary artery obstruction which leads to increased pulmonary vascular resistance and consequently, a right ventricle resistance. Increased right ventricle resistance can cause an acute right ventricle dilatation with hypokinesia and severe myocardial ischemia all leading to increase the troponin levels [4]. Other mechanisms that could explain elevated troponins in PE include: reduced coronary perfusion, hypoxemia from perfusion-ventilation mismatch, systemic hypo perfusion, or multiple of these mechanisms [5]. cTnT may improve risk stratification in patients with PE and may aid in the identification of patients in whom a more aggressive therapy may be warranted.

Cardiac troponin I (cTnI) has a role in risk stratification and short-term prognostication in patients with acute pulmonary embolism [6]. Increased serum cTnI levels is a marker for severe PE in patients who present with electrocardiographic (EKG) and echocardiographic features suggesting pulmonary embolism [7]. Konstantinides et al. [8] suggested that either elevate cTnI alone or elevated cTnI with EKG changes and clinical findings for right ventricle dysfunction could be used to evaluate the right ventricle dysfunction. In fact, up to $70.4 \%$ of patients who have a PE and 
elevated cTnI were found to have right ventricular dysfunction (RVD) $[7,9]$. In a prospective multi-centre trial including 90 patients with confirmed acute PE from 12 collaborating hospitals, Zhu et al. [10] found that cTnI is an independent predictor of 14 day clinical outcomes for patients with a PE. In addition, they noted that RVD combined with cTnI can identify a subgroup of acute PE patients with a much more guarded prognosis [10] Echocardiographic evaluation demonstrated that most of the patients with high cTnI values developed RVD and are at higher risk of developing cardiogenic shock $[6,9]$.

A number of studies found higher cTnI levels in sub-massive PE patients compared with non-massive PE patients [12]. Kilinc et al. [7] suggested that high cTnI was higher in massive PE compared with sub-massive and non-massive PE [7]. An increase in both cTnI and D-dimer have a sensitivity of more than $90 \%$ for the diagnosis of PE but the specificity is low because other respiratory and cardiologic conditions also have elevated cTnI and D-dimer levels [7].

Elevated cardiac troponins in PE are associated with the severity and the prognosis of the disease rather than diagnosis $[13,14]$. EKG evidence of S1Q3T3 pattern and ST-T wave changes are the most frequent pathologic EKG findings seen in PE patients with high cTnI [7]. In fact, patients who had PE with elevated cTnI levels more frequently had S1Q3T3 pattern, right bundle branch block and T wave changes compared to patients with normal cTnI level [14]. RVD was found in $70.4 \%$ of the patients with high cTnI and $57.7 \%$ of the patients with normal cTnI [8].

Elevated cTn levels were significantly associated with shortterm mortality, resulting from PE and other adverse events [8]. Increased cTn values were also associated with a higher mortality in the subgroup of hemodynamically stable patients [8]. Jime'nez D et al. [15] completed a meta-analysis focused on normotensive patients with acute symptomatic PE. This analysis consisting of 1366 patients revealed that elevated troponin levels resulted in a four-fold increased risk of short-term death [15].

\section{End Stage Renal Disease (ESRD)}

Serum concentrations of the cardiac troponins (cTn) $\mathrm{T}$ and I, specific markers of myocardial injury, are frequently elevated in hemodialysis patients [17]. (Mc Lauren) The prevalence of ischemic heart disease (IHD) in hemodialysis patients is $10-20$ times higher than that in the general population. Cardiovascular disease causes $50 \%$ mortality in patients on hemodialysis and $42 \%$ of patients undergoing hemodialysis have had a myocardial infarction or coronary revascularization. (Marie) Serum cardiac troponin $\mathrm{T}$ (cTnT) concentrations are frequently increased in chronic dialysis patients [17]. The clinical symptoms of cardiac damage are difficult to diagnose in patients on hemodialysis and so it is unclear how high troponin levels should be interpreted [18]. The increase in serum troponin levels in dialysis patients may be due to subclinical myocardial injury and/or abnormalities of troponins catabolism induced by renal failure and even hemodialysis itself.

Cardiomyopathy of hemodialysis patients may explain elevated cTn levels in these patients. This is due to microvascular lesions or direct injury to myocardial cells (e.g. toxic, stretching, hypoxia, apoptosis) that are encountered in the cardiomyopathy of hemodialysis patients. A study completed by Marie Christine Iliou et al. [19] shows that elevated cTn concentrations are observed in the basal state in a non-negligible percentage of chronic hemodialysis patients. A reported $18 \%-53 \%$ of patients have elevated cTn before dialysis [19,20]. Most of these patients also have diabetes and diabetes is associated with the development of microangiopathy and myocardial ischemia, which may be clinically silent which may explain the increase in cTn levels.

The kinetics of decrease and the catabolic pathways of cTn in hemodialysis patients are not known. Some evidence suggests that $\mathrm{cTnI}$ is fragmented into pieces of $8-25 \mathrm{kD}$ that are normally released in very small quantities in the blood and are sufficiently small enough to be cleared by the healthy kidney. In patients with ESRD, these microfragments cannot be cleared from blood and are detectable by immunoassays [5]. Little is known about the route of elimination of cTn. Hemolysis may interfere with cTn and cause measurement of higher or lower cTn concentrations.

Urinary clearance rates of less than $0.01 \mathrm{ml} / \mathrm{min}$ for cTnT and of less than $1.15 \mathrm{ml} / \mathrm{min}$ for $\mathrm{cTnI}$ have been reported in a patient without renal impairment following acute myocardial infarction [21]. Studies have reported a positive correlation between the serum concentrations of creatinine and cTn in chronic renal failure patients before hemodialysis [22], and a negative relationship between the serum levels of cTn and creatinine in hemodialysis patients [23].

Elevated serum levels of cTn are observed in 5.0-20.0\% of chronic hemodialysis patients without acute symptoms [16]. Given the elevated serum levels of cTn in a high proportion of asymptomatic patients, interpretation of slightly elevated serum levels of cTn in hemodialysis patient with acute chest pain, need to take baseline concentration into consideration. Very high serum levels of cTn with acute symptoms and/or EKG changes must be interpreted as a myocardial infarction, as in normal renal function patients.

\section{Sepsis/ Systemic Inflammatory Response Syndrome (SIRS) and Critically Ill Patient}

Elevated cardiac troponin I (cTnI) is frequently observed in patients with severe sepsis and septic shock. However, the mechanisms underlying cTnI release in these patients are still unknown. Proposed mechanisms are cytokine-mediated reversible myocardial membrane leakage and/or apoptosis [24]. Elevated cTn have been detected in $36 \%$ (cTnT $\geqslant 0.1 \mathrm{ng} /$ $\mathrm{ml}$ ) to $85 \%$ (cTnI $>0.1 \mathrm{ng} / \mathrm{ml}$ ) of patients who are treated in intensive care units for sepsis or systemic inflammatory response syndrome (SIRS) [24]. Cardiovascular dysfunction is pronounced in patients with severe sepsis and is characterized by elements of hypovolemic, cytotoxic, and distributive shock. Coronary artery disease is hardly the cause of cTn elevation in these patients, since coronary artery disease is usually ruled out. So elevation in troponin is likely caused by other clinical conditions. Clinical and experimental studies suggest that cytokines, especially TNF $\alpha$, IL-1 $\beta$ and IL- 6 appear to play a pivotal role in mediating the hemodynamic effects and the release of cardiac troponin in 
patients with severe sepsis and septic shock [24]. Oxygen supply and demand is one of the most widely agreed mechanisms for troponin increase.

A mismatch in oxygen supply and demand of the myocardium could be a reason for the release of cTn from damaged myocardial cells. As a consequence of fever and tachycardia the oxygen demand of the myocardium is increased. Simultaneously, oxygen supply to the myocardium is reduced due to systemic hypoxemia from respiratory failure, microcirculatory dysfunction, hypotension and sometimes anemia [24].

Elevated cTn values provide prognostic information and the extent of cTn elevation seems to correlate with the severity of the disease process. Several studies have demonstrated that the presence of elevated troponin levels in critically ill septic patients predict the presence of myocardial dysfunction and an increased mortality rate $[25,26]$. A significant correlation between the serum level of cTnI and the reduction in ejection fraction has also been noted [11]. Ver Elst et al. [27] demonstrated that cTnI positivity was strongly associated with left ventricle dysfunction (LVD) $(78 \%$ v 9\% in cTnI negative patients; $p<0.001)$ [27]. cTnl values were found to correlate significantly with the degree of hypotension and APACHE II score [24]. Spies et al. [24] also found out in their study of 26 septic patients that patients with cTnT values of $0.2 \mu / \mathrm{l}$ or higher had an increased mortality rate $(83 \%$ $\mathrm{v} 38 \%, \mathrm{p}=0.02$ ) compared to the group with cTnT values below this value. Elevated troponins in the critically ill is associated with an adverse prognosis irrespective of the underlying cause [28]. Sepsis induced myocardial dysfunction may reflect cardiac hibernation and be an adaptive response, so judicious use of catecholamines is recommended. Adrenergic stimulation leads to an increase in cardiac work and may damage cardiomyocytes [29].

\section{Rhabdomyolysis}

Rhabdomyolysis is a condition of skeletal muscle injury caused by trauma, pharmaceutical agents (benzodiazepines, neuroleptics, and anti-depressives), alcohol, illicit drugs and critical illness $[30,31]$. Myocyte injury leads to an increase in cytoplasmic calcium which activates reactions that result in cell lysis and release of cellular contents such as myoglobin, creatinine kinase (CK), uric acid etc [30]. Troponin elevations during rhabdomyolysis occur in 11-30\% of cases [32-34]. Punukollu et al. [33] examined the etiology and clinical significance of troponin elevations in hospitalized patients with $\mathrm{CK}>1000 \mathrm{U} / \mathrm{L}$. Troponin elevations were found in patients with heavy resistance exercise, chronic myopathy, and traumatic and non-traumatic rhabdomyolysis [33]. Patients with the cTnI elevation were found to be sicker associated with increased rates of ICU admission, hypotension, sepsis and length of stay compared to those without troponin elevation [33]. A retrospective study of emergency room patients with $\mathrm{CK}>1000 \mathrm{U} / \mathrm{L}$ and $\mathrm{CTnI} \geq 0.1 \mu \mathrm{g} / \mathrm{L}$ evaluated the significance of the troponin elevation in patients with rhabdomyolysis. Troponin elevation was determined to be a false positive if the patient had normal echocardiography and EKG. The study found that $50 \%$ of the cohort had a troponin elevation, one-third of that $50 \%$ were false positives (17\%) [32]. The author concludes that the false positive troponin may represent minor cardiac injuries or an inherent flaw of the assay that may identify cross-reactions with skeletal muscle proteins [32].

A case report of a 27 year old man with rhabdomyolysis secondary to amphetamine use and incarcerated hernia demonstrated two elevations of troponin levels: 3 days and 9 days post-operatively, with maximum on post-operative day 18 [35]. The authors attributed this peak in cTnT and not cTnI on day 9 as possible re-expression of cTnT, an isoform during skeletal muscle regeneration $[35,36]$. However, the mechanism of troponin rise in rhabdomyolysis, remains poorly understood and debated [35,37]. As demonstrated in the literature, troponin elevation is common among patients with rhabdomyolysis and may portend poor patient outcomes [32-34]. Treatment of rhabdomyolysis with aggressive fluid hydration can improve troponin elevation back to baseline [36]. Still, a cardiac work-up is recommended to evaluate patients with risk factors for coronary artery disease (CAD) after resolution of rhabdomyolysis [38].

\section{Physical Exercise}

The cardiovascular benefits of regular physical exercise have been well established. Prolonged extreme exercise may cause elevations in cTn levels and has historically been a clinical conundrum due to its association with "cardiac fatigue" and transiently decreased systolic and diastolic function [39]. There are few studies with long term follow up demonstrating no harm secondary to this cardiac stress. Notwithstanding the lack of evidence, case reports have associated right ventricular dysplasia and reduced ejection fraction in endurance athletes [40].

Release of troponin $(0.02$ to $0.04 \mu \mathrm{g} / \mathrm{L})$ begins as early as 30 min of sustained exercise [41]. A study of adolescent and adult athletes demonstrated increases in post exercise troponin levels after only 90 minutes of treadmill running [42]. Troponin levels peaked at 3-4 hours [42]. Participants also demonstrated immediate reduction in left ventricular ejection fraction (LVEF) and doppler flow velocities. Both metrics returned to baseline at 6 hours post exercise. The study found no correlation between troponin elevation and these outcomes [42].

Studies have shown increases in serum troponin levels of $80-100 \%$ of marathon runners post-race $[39,43]$. The variation in troponin elevation may be related to baseline fitness of participants, time of testing and exercise type, duration, and intensity [44]. A meta-analysis showed higher elevations in runners compared to cyclers and traiathloners [41]. A study of marathon runners by Eijsvogels et al. demonstrates elevations in the resting, post-marathon and post $24 \mathrm{hrs}$, for both contemporary and high sensitivity cTnI asseys from (13\%, 78\% and 25\%) to (99\%,100\%, and 27\%) respectively [43].Other studies have even shown increases in troponin after prolonged walking in nonathletes [45].

Exercise induced troponin elevation is poorly understood. Suggested mechanism includes myocardial stunning and transient changes in membrane permeability related to elevated reactive oxygen, a species production that occurs with prolonged exercise. Troponin rise can be an indication of cardiac injury and regeneration of cardiac myocytes [46]. 


\section{Burns}

In 2008, the World Health Organization estimated that over 3 million people die from fire-related burns worldwide [9]. The effects of burns on cardiac function have been demonstrated in animal models, however, have been scarcely published on humans. Troponin leakage is thought to be secondary to the inflammatory and hypoxic effects on myocytes [10]. Observational studies have shown that patients with greater burn total body surface (TBSA) are at higher risk for myocardial damage reflected by increased troponin levels. An observational study of 30 burn patients found a correlation between troponin rise and severity of clinical outcomes [11]. Troponin levels were measured on day 5 and at 2 day intervals until day 14. Patients with total body surface area of burn $>30 \%$ had significantly higher cTnI rise compared to those with less burn area [11]. Troponin level peaked at day 9 correlating with the development of wound infection and exudation. In one patient's case with delayed fluid resuscitation, troponin levels peaked earlier with more persistent elevations [11]. Murphy et al. [12] found that in twenty-three patients cTnI was detectable at 3 hours for patients with burn TBSA> 25\% and peaked at 12 hours [12]. Bak et al. [10] evaluated cardiac dysfunction in a study of 10 consecutive patients with burn TBSA $>20 \%$ who required mechanical ventilation. Cardiac dysfunction was measured by serial transesophageal echocardiogram (TEE) and serologic troponin and transaminase levels measured at 12, 24, and 36 hours post-burn. Evidence of cardiac wall motion abnormalities on TEE correlated with a rise in troponin. All patients in the study with normal troponin levels also had normal echocardiograms with no evidence of wall motion abnormalities [10]. Current evidence suggests the utility of serological troponin in risk stratifying burn patients. Increased troponins may predict patients at risk for wound infection occurrence and cardiac dysfunction and may also serve as a marker of fluid resuscitation efficacy.

\section{Drug Toxicity}

Baseline troponin elevations have been seen in patients with hematologic malignancies [47]. Subsequent treatments with chemotherapy may cause further rise in serum troponin levels. Cardiotoxicity due to drugs has been largely attributed to chemotherapy. Anthracyclines play a major role in the treatment of many cancers including breast, lymphoma and other childhood malignancies [48]. Doxorubicin, a commonly used anthracycline has been effective against neoplasms; however dose dependent cardiomyopathy limits its use at high doses [49]. Anthracycline induced DNA damage, due to reactive oxygen species production, are thought to cause cardiomyocyte apoptosis and myofibril degradation [48]. The improvement in sensitivity of cardiac troponin tests have increased their utility as early predictors of cardiotoxicity for chemotherapy patients [50].

Patients with cancer can experience adverse cardiovascular events secondary to the malignant process itself or its treatment. Chemotherapeutic agents such as anthracyclines, alkylating agents, anti-metabolites or anti-microtubules have cardiotoxic side effects. These chemotherapeutic agents have been associated with side effects such as ischemia from anti metabolites, cardiomyopathy, pericarditis, endomyocardial fibrosis, and arrhythmias [5]. Several oncological treatment regimens incorporate multiple agents whose deleterious cardiac effects may be additive or even synergistic. In addition, with increase in life expectancy, cancer patients also have comorbidities including underlying cardiovascular disease which may be exacerbated by the stress of the tumor growth or its treatment. Troponins and natriuretic peptides could be useful in identifying patients at risk for myocardial damage and in monitoring the development of cardiac damage. These markers could someday be used to influence chemotherapeutic choices.

A third of hematologic and breast cancer patients treated with high dose anthracycline have increases in troponin levels [47]. Similarly, an early rise in troponin during transtuzamab was associated with 17.6 times increased risk of cardiotoxicity [51]. These changes are thought to precede those seen by echocardiography [47]. The risk of cardiotoxicity and LVD persist even after the cessation of therapy [47]. Studies have demonstrated that cancer patients with persistent elevations have higher likelihood and magnitude of LVD compared with those who have transient troponin elevation $[47,52,53]$. Administration of 5-FU has also been associated clinically insignificant cTnI elevation [49].

In a study of 43 Herceptin- 2 positive breast cancer patients, troponin elevation at 3-months post anthracycline/transtuzamab or transtuzamab only treatment was shown to independently predict cardiotoxicity at 6months [53]. Cardinale et al. [48] conducted a randomized controlled trial of 473 cancer patients receiving high dose chemotherapy to evaluate the cardioprotective effects of enalapril in reducing cardiotoxicity [48]. Patients with a troponin I level of $>0.07 \mathrm{ng} / \mathrm{ml}$ were considered for enrollment and randomized to enalapril or control group [52]. Cardiotoxicity was defined using the international oncological guidelines as an absolute reduction in LVEF $>10 \%$ resulting in LVEF lower than the normal limit value. Forty three percent of patients in the control group demonstrated a reduction in LVEF compared to none in the enalapril group. Additionally, the number of patients showing an increased troponin level during the follow up period was higher than those in the ACEI group. Thus, early treatment with enalapril soon after high dose chemotherapy appears to be cardio-protective and prevent cardiotoxicity and its sequela. These findings represent the diagnostic utility of cardiac troponins of predicting those most at risk of cardiotoxicity and implementing interventions to halt the development of cardiac dysfunction and negative sequela [52]. Patients receiving high dose chemotherapy without troponin elevation had no cardiac events reported at 1 year follow up [52].

Tocolytic drugs Fenoterol and Verapamil increased troponin level in a prospective cohort study of 22 women. Thirty-two percent and $40 \%$ of women had troponin elevation at day 1 and day 3 of treatment, respectively [54]. These medications modify cellular membrane permeability, leading to increased cellular metabolism, cardiac activity and myonecrosis [54]. Cocaine has also been associated with cardiotoxic effects caused by vasoconstriction and increased cardiac sympathetic stimulation. These effects may be demonstrated by troponin rise [49]. 
Colchicine, medication commonly used for the treatment of gout flare has been associated with troponin elevation. Two case reports documenting suicidal attempts with high colchicine consumption showed elevated troponin levels prior to cardiovascular collapse. Both patients had sustained rise of troponin up to 4 days post ingestion [55,56].

Following overdose, myocardial injury is common [57]. An observational study of 625 patients presenting to the ED for suspected drug overdose, found that the use of cTnI was an independent predictor of mortality. Benzodiazepines, opiods, sympathomimetics, acetaminophen and antidepressants were the five most commonly overdosed drugs used. Even after sensitivity analysis for $\mathrm{CAD}$ and adjusting for age and gender, troponin elevation remained an independent predictor of mortality [58]. A normal troponin level had a negative predictive value of $>99 \%$ for ruling out fatality [58]. These findings highlight the diagnostic utility of troponin levels at risk stratifying patients with drug overdose and at risk for toxicity.

\section{Stroke}

Cardiovascular and cerebrovascular diseases are closely associated with shared risk factors, pathogenesis and preventive strategies. Patients with cerebrovascular disease are at increased risk for cardiovascular events and are in fact the $2^{\text {nd }}$ cause of death in those presenting with an acute stroke. This high prevalence of cardiovascular disease has led the professional societies, American Heart Association and American Stroke Association, to include troponin evaluation in the recommended guidelines for all patients presenting with acute ischemic stroke (AIS) [59].

There is no clearly defined mechanism of troponin elevation in AIS. Proposed mechanisms include neurologic myocardial damage, emotional or physical stress, which increases catecholamine release altering the autonomic function [60]. This alteration in autonomic dysfunction may elicit a type II MI by causing vasoconstriction and/or reduction in blood flow to coronary vessels with stable plaques $[60,61]$. Others suggest that the exaggerated release of catecholamine increase calcium permeability of myocytes causing reversible myocyte dysfunction [61].

The clinical significance of troponin elevation in the setting of AIS has been widely studied and has been associated with increased morbidity and mortality. Non-acute MI occurs in 20 $60 \%$ of those with AIS, moreover silent CAD may contribute to future cardiac morbidity and mortality [62,63]. Amarenco et al. [64] found that $26 \%$ of AIS patients without known cardiac history had asymptomatic obstructive CAD [64]. A retrospective analysis of patients hospitalized for AIS found that $85 \%$ of patients had undergone troponin testing, $21 \%$ had troponin elevation and $10 \%$ had echocardiogram findings suggestive of cardiac dysfunction. Of the patients with elevated troponin levels 35\% had no prior history of CAD [62]. Remarkably troponin elevation was associated with $26 \%$ mortality at 30 days, $47 \%$ at 1 year and $60 \%$ at 3 years [62].

Anders et al. [65] found that in a prospective trial of AIS, there was significant variation in the incidence of troponin elevation
$(>0.045 \mathrm{ng} / \mathrm{mL})$ in the stroke subtypes: $21 \%$ of ischemic stroke, $34 \%$ of intracranial hemorrhage and $7 \%$ of transient ischemic attacks [65]. Hays et al. [66] reported troponin elevations in $14 \%$ of patients with ischemic stroke, $15 \%$ of intracerebral hemorrhage and $21 \%$ of subarachnoid hemorrhage [66]. Compared to those with normal troponin in-hospital mortality was found be statistically significant in ischemic stroke, intracerebral and subarachnoid hemorrhage [66]. Patients with an elevated troponin have demonstrated increased rates of stroke risk factors (hypertension, atrial fibrillation), CHF,CKD and MI $[63,65]$. In a prospective control-matched observational study of patients admitted with AIS and NSTEM (controls), found that AIS patients had less CAD despite similar troponin levels compared to the controls [63]. LVEF and Takotsubo cardiomyopathy were similar between the two populations [63].

Liu etal. [67] found a link between hemorrhagic transformation in ischemic stroke patients with rheumatologic disease and troponin elevation [67]. A small prospective study of Chines patients with acute stroke patients with atrial fibrillation and/ or rheumatologic disease and high sensitive troponin levels > $17.78 \mathrm{ng} / \mathrm{L}$ (reference range $0-14 \mathrm{ng} / \mathrm{L}$ ) and cerebral micro bleeds (CMB) [68]. This finding remained after adjusting for co-variates [68]. Troponin elevations have also been shown to be more prevalent in specific stroke locations. Infact, Lasek-Bal found more frequent $\mathrm{R}$ hemisphere strokes [69].

The RE-LY trial showed a significantly higher risk of stroke for patients with persistent troponin elevation and atrial fibrillation compared with those with transient elevations [70]. TRELAS subanalysis of AIS patients without a history of or EKG evidence of atrial fibrillation on admission showed that patients with higher hs-cTnT was associated with atrial fibrillation on in-hospital EKG monitoring [71]. The study determined that a troponin level of $17 \mathrm{ng} / \mathrm{L}$ (sensitivity $50 \%$; specificity $74.2 \%$ ) differentiated patients with and without new pAF [71].

Troponin levels in the setting of stroke have been shown to prognosticate short and long term morbidity and mortality [71]. Screening for CAD is recommended for those with persistent and dynamic elevations to prevent future adverse events $[63,71]$.

\section{Conclusion}

Cardiac troponins $\mathrm{T}$ and I have typically been used for the diagnosis and prognosis of acute coronary syndrome (ACS) but are elevated in several other clinical conditions. Although not all the mechanisms for the troponin elevation are known, in some conditions it can be attributed to reduced oxygen supply to the myocardium. This can be seen in clinical settings such as sepsis, the systemic inflammatory response syndrome, hypotension or hypovolemia, critically ill patients and tachyarrhythmias [72-75]. Since type II myocardial ischemia (MI) and myocardial injury are associated with poor outcomes in both the short term and long term, it is important to recognize these entities as soon as possible to identify higher risk patients. This article presents evidence that highlights the importance of elevated troponins in the absence of ACS. Further studies and guidelines are needed to help clinicians appropriately manage patients with type II MI and non-ischemic 
myocyte injury so that we can continue to decrease the significant morbidity and mortality associated with myocardial injury.

\section{References}

1. Rubini Gimenez M, Twerenbold R, Reichlin T, Karin W, Philip H, et al. (2014) Direct comparison of high-sensitivity-cardiac troponin I vs. $\mathrm{T}$ for the early diagnosis of acute myocardial infarction. Eur Heart J 35(34): 2303-2311.

2. Lankeit M, Friesen D, Aschoff J, Dellas C, Hasenfuss G, et al. (2010) Highly sensitive troponin $\mathrm{T}$ assay in normotensive patients with acute pulmonary embolism. Eur Heart J 31(15): 1836-1844.

3. Giannitsis E, Muller-Bardorff M, Lehrke S, Wiegand U, Tölg R, et al. (2001) Admission troponin T level predicts clinical outcomes, TIMI flow, and myocardial tissue perfusion after primary percutaneous intervention for acute ST-segment elevation myocardial infarction. Circulation 104(6): 630-635.

4. Lualdi JC, Goldhaber SZ (1995) Right ventricular dysfunction after acute pulmonary embolism: pathophysiologic factors, detection, and therapeutic implications. Am Heart J 130(6): 1276-1282.

5. Korff S, Katus HA, Giannitsis E (2006) Differential diagnosis of elevated troponins. Heart 92(7): 987-993.

6. Mehta NJ, Jani K, Khan IA (2003) Clinical usefulness and prognostic value of elevated cardiac troponin I levels in acute pulmonary embolism. Am Heart J 145(5): 821-825.

7. Kilinc G, Dogan OT, Berk S, Epozturk K, Ozsahin SL, et al. (2012) Significance of serum cardiac troponin I levels in pulmonary embolism. J Thorac Dis 4(6): 588-593.

8. Konstantinides S, Geibel A, Olschewski M, Kasper W, Hruska N, et al. (2002) Importance of cardiac troponins I and T in risk stratification of patients with acute pulmonary embolism. Circulation 106(10): 1263-1268.

9. Meyer T, Binder L, Hruska N, Luthe H, Buchwald AB (2000) Cardiac troponin I elevation in acute pulmonary embolism is associated with right ventricular dysfunction. J Am Coll Cardiol 36(5): 16321636.

10. Zhu L, Yang YH, Wu YF, Zhai ZG, Wang C, et al. (2007) Value of transthoracic echocardiography combined with cardiac troponin I in risk stratification in acute pulmonary thromboembolism. Chin Med J (Engl) 120(1): 17-21.

11. Mehta NJ, Khan IA, Gupta V, Jani K, Gowda RM, et al. (2004) Cardiac troponin I predicts myocardial dysfunction and adverse outcome in septic shock. Int J Cardiol 95(1): 13-17.

12. Amorim S, Dias P, Rodrigues RA, Araújo V, Macedo F, et al. (2006) Troponin $I$ as a marker of right ventricular dysfunction and severity of pulmonary embolism. Rev Port Cardiol 25(2): 181-186.

13. Becattini C, Vedovati MC, Agnelli G (2007) Prognostic value of troponins in acute pulmonary embolism: a meta-analysis. Circulation 116(4): 427-433.

14. Janata K, Holzer M, Laggner AN, Mullner M (2003) Cardiac troponin $\mathrm{T}$ in the severity assessment of patients with pulmonary embolism: cohort study. BMJ 326(7384): 312-313.

15. Jimenez D, Uresandi F, Otero R, Lobo JL, Monreal M, et al. (2009) Troponin-based risk stratification of patients with acute nonmassive pulmonary embolism: systematic review and metaanalysis. Chest 136(4): 974-982.
16. Iliou MC, Fumeron C, Benoit MO, Tuppin P, Calonge VM, et al. (2003) Prognostic value of cardiac markers in ESRD: Chronic Hemodialysis and New Cardiac Markers Evaluation (CHANCE) study. Am J Kidney Dis 42(3): 513-523.

17. McLaurin MD, Apple FS, Voss EM, Herzog CA, Sharkey SW (1997) Cardiac troponin I, cardiac troponin T, and creatine kinase MB in dialysis patients without ischemic heart disease: evidence of cardiac troponin $\mathrm{T}$ expression in skeletal muscle. Clin Chem 43(6 Pt 1): 976-982.

18. Jaffe AS, Ritter C, Meltzer V, Harter H, Roberts R (1984) Unmasking artifactual increases in creatine kinase isoenzymes in patients with renal failure. J Lab Clin Med 104(2): 193-202.

19. Ooi DS, House AA (1998) Cardiac troponin $T$ in hemodialyzed patients. Clin Chem 44(7): 1410-1416.

20. Roppolo LP, Fitzgerald R, Dillow J, Ziegler T, Rice M, et al. (1999) A comparison of troponin $\mathrm{T}$ and troponin I as predictors of cardiac events in patients undergoing chronic dialysis at a Veteran's Hospital: a pilot study. J Am Coll Cardiol 34(2): 448-454.

21. Willging S, Keller F, Steinbach G (1998) Specificity of cardiac troponins I and T in renal disease. Clin Chem Lab Med 36(2): 8792.

22. Collinson PO, Boa FG, Gaze DC (2001) Measurement of cardiac troponins. Ann Clin Biochem 38(Pt 5): 423-449.

23. Lowbeer C, Gutierrez A, Gustafsson SA, Norrman R, Hulting J, et al. (2002) Elevated cardiac troponin T in peritoneal dialysis patients is associated with CRP and predicts all-cause mortality and cardiac death. Nephrol Dial Transplant 17(12): 2178-2183.

24. Spies C, Haude V, Fitzner R, Schröder K, Overbeck M, et al. (1998) Serum cardiac troponin $\mathrm{T}$ as a prognostic marker in early sepsis Chest 113(4): 1055-1063.

25. Parmacek MS, Solaro RJ (2004) Biology of the troponin complex in cardiac myocytes. Prog Cardiovasc Dis 47(3): 159-176.

26. Dokainish H, Pillai M, Murphy SA, DiBattiste PM, Schweiger MJ, et al. (2005) Prognostic implications of elevated troponin in patients with suspected acute coronary syndrome but no critical epicardial coronary disease: a TACTICS-TIMI-18 substudy. J Am Coll Cardiol 45(1): 19-24.

27. ver Elst KM, Spapen HD, Nguyen DN, Garbar C, Huyghens LP, et al. (2000) Cardiac troponins I and T are biological markers of left ventricular dysfunction in septic shock. Clin Chem 46(5): 650-657.

28. King DA, Codish S, Novack V, Barski L, Almog Y (2005) The role of cardiac troponin I as a prognosticator in critically ill medical patients: a prospective observational cohort study. Crit Care 9(4): R390-395.

29. Singer M (2007) Catecholamine treatment for shock--equally good or bad? Lancet 370(9588): 636-637.

30. Zimmerman JL, Shen MC (2013) Rhabdomyolysis. Chest 144(3): 1058-1065.

31. Lofberg M, Tahtela R, Harkonen M, Somer H (1996) Cardiac troponins in severe rhabdomyolysis. Clinical chemistry 42(7): 1120-1121.

32. Li SF, Zapata J, Tillem E (2005) The prevalence of false-positive cardiac troponin I in ED patients with rhabdomyolysis. Am J Emerg Med 23(7): 860-863. 
33. Punukollu G, Gowda RM, Khan IA, Mehta NJ, Navarro V, et al. (2004) Elevated serum cardiac troponin I in rhabdomyolysis. Int J Cardiol 96(1): $35-40$

34. Lofberg M, Tahtela R, Harkonen M, Somer H (1995) Myosin heavy-chain fragments and cardiac troponins in the serum in rhabdomyolysis. Diagnostic specificity of new biochemical markers. Arch Neurol 52(12): 1210-1214.

35. Sribhen K, Phankingthongkum R, Wannasilp N (2012) Skeletal muscle disease as noncardiac cause of cardiac troponin T elevation. J Am Coll Cardiol 59(14): 1334-1335.

36. Jaffe AS, Vasile VC, Milone M, Saenger AK, Olson KN, et al. (2011) Diseased skeletal muscle: a noncardiac source of increased circulating concentrations of cardiac troponin T. J Am Coll Cardiol 58(17): 1819-1824.

37. Giannitsis E, Katus HA (2012) Challenging interpretation of elevated cardiac troponin $\mathrm{T}$ in a complex case with rhabdomyolysis. J Am Coll Cardiol 60(11): 1027-1028.

38. Nobre C, Thomas B (2014) Elevation of troponins in rhabdomyolysis. Chest 145(2): 415.

39. Agewall S, Giannitsis E, Jernberg T, Katus H (2011) Troponin elevation in coronary vs. non-coronary disease. Eur Heart J 32(4): 404-411.

40. Harper RW (2010) Exercise-induced troponin elevation: not necessarily a benign phenomenon. J Am Coll Cardiol 56(25): 2145

41. Shave R, George KP, Atkinson G, Hart E, Middleton N, et al. (2007) Exercise-induced cardiac troponin T release: a meta-analysis. Med Sci Sports Exerc 39(12): 2099-2106.

42. Tian Y, Nie J, Huang C, George KP (2012) The kinetics of highly sensitive cardiac troponin $\mathrm{T}$ release after prolonged treadmill exercise in adolescent and adult athletes. J Appl Physiol (1985) 113(3): 418-425.

43. Eijsvogels TM, Shave R, van Dijk A, Hopman MT, Thijssen DH (2011) Exercise-induced cardiac troponin release: real-life clinica confusion. Curr Med Chem 18(23): 3457-3461.

44. Shave R, Baggish A, George K, Wood M, Scharhag J, et al. (2010) Exercise-induced cardiac troponin elevation: evidence, mechanisms, and implications. J Am Coll Cardiol 56(3): 169-176.

45. Eijsvogels T, George K, Shave R, Gaze D, Levine BD, et al. (2010) Effect of prolonged walking on cardiac troponin levels. Am Cardiol 105(2): 267-272.

46. Jaffe AS (2012) Troponin--past, present, and future. Curr Probl Cardiol 37(6): 209-228.

47. Christenson ES, James T, Agrawal V, Park BH (2015) Use of biomarkers for the assessment of chemotherapy-induced cardiac toxicity. Clin Biochem 48(4-5): 223-235.

48. McGowan JV, Chung R, Maulik A, Piotrowska I, Walker JM, et al. (2017) Anthracycline Chemotherapy and Cardiotoxicity. Cardiovasc Drugs Ther 31(1): 63-75.

49. Gaze DC, Collinson PO (2005) Cardiac troponins as biomarkers of drug- and toxin-induced cardiac toxicity and cardioprotection. Expert Opin Drug Metab Toxicol 1(4): 715-725.

50. Roziakova L, Mistrik M, Batorova A, Kruzliak P, Bojtarova E, et al. (2015) Can we predict clinical cardiotoxicity with cardiac biomarkers in patients after haematopoietic stem cell transplantation? Cardiovasc Toxicol 15(3): 210-216.
51. Cardinale D, Colombo A, Torrisi R, Sandri MT, Civelli M, et al. (2010) Trastuzumab-induced cardiotoxicity: clinical and prognostic implications of troponin I evaluation. J Clin Oncol 28(25): 39103916.

52. Cardinale D, Colombo A, Sandri MT, Giuseppina L, Nicola C, et al. (2006) Prevention of high-dose chemotherapy-induced cardiotoxicity in high-risk patients by angiotensin-converting enzyme inhibition. Circulation 114(23): 2474-2481.

53. Sawaya H, Sebag IA, Plana JC, Januzzi JL, Ky B, et al. (2011) Early detection and prediction of cardiotoxicity in chemotherapytreated patients. Am J Cardiol 107(9): 1375-1380.

54. Adamcova M, Gersl V, Hrdina R, Melka M, Mazurová Y, et al. (1999) Cardiac troponin $\mathrm{T}$ as a marker of myocardial damage caused by antineoplastic drugs in rabbits. J Cancer Res Clin Oncol 125(5): 268-274.

55. Mullins ME, Robertson DG, Norton RL (2000) Troponin I as a marker of cardiac toxicity in acute colchicine overdose. Am J Emerg Med 18(6): 743-744.

56. van Heyningen C, Watson ID (2005) Troponin for prediction of cardiovascular collapse in acute colchicine overdose. Emerg Med J 22(8): 599-600.

57. Manini AF, Nelson LS, Stimmel B, Vlahov D, Hoffman RS (2012) Incidence of adverse cardiovascular events in adults following drug overdose. Acad Emerg Med 19(7): 843-849.

58. Manini AF, Stimmel B, Hoffman RS, Vlahov D (2016) Utility of Cardiac Troponin to Predict Drug Overdose Mortality. Cardiovasc Toxicol 16(4): 355-360.

59. Jauch EC, Saver JL, Adams HP, Bruno A, Connors JJ, et al. (2013) Guidelines for the early management of patients with acute ischemic stroke: a guideline for healthcare professionals from the American Heart Association/American Stroke Association. Stroke 44(3): 870-947.

60. Scheitz JF, Nolte CH, Laufs U, Endres M (2015) Application and interpretation of high-sensitivity cardiac troponin assays in patients with acute ischemic stroke. Stroke 46(4): 1132-1140.

61. Jensen JK, Ueland T, Aukrust P, Antonsen L, Kristensen SR, et al (2012) Highly sensitive troponin T in patients with acute ischemic stroke. European neurology 68(5): 287-293.

62. Wrigley P, Khoury J, Eckerle B, Alwell K, Moomaw CJ, et al Prevalence of Positive Troponin and Echocardiogram Findings and Association With Mortality in Acute Ischemic Stroke. Stroke 48(5): 1226-1232.

63. Mochmann HC, Scheitz JF, Petzold GC, Haeusler KG, Audebert HJ et al. (2016) Coronary Angiographic Findings in Acute Ischemic Stroke Patients With Elevated Cardiac Troponin: The Troponin Elevation in Acute Ischemic Stroke (TRELAS) Study. Circulation 133(13): 1264-1271.

64. Amarenco P, Lavallee PC, Labreuche J, Ducrocq G, Juliard JM, et al. (2011) Prevalence of coronary atherosclerosis in patients with cerebral infarction. Stroke 42(1): 22-29.

65. Anders B, Alonso A, Artemis D, Schäfer A, Ebert A, et al. (2013) What does elevated high-sensitive troponin I in stroke patients mean: concomitant acute myocardial infarction or a marker for high-risk patients? Cerebrovasc Dis 36(3): 211-217.

66. Hays A, Diringer MN (2006) Elevated troponin levels are associated with higher mortality following intracerebral hemorrhage. Neurology 66(9): 1330-1334. 
67. Liu X, Chen X, Wang H, Pan S (2014) Prognostic significance of admission levels of cardiac indicators in patients with acute ischaemic stroke: prospective observational study. J Int Med Res 42(6): 1301-1310.

68. Liu J, Wang D, Xiong Y, Liu B, Wei C, et al. (2016) High-sensitivity cardiac troponin $\mathrm{T}$ levels and risk of cerebral microbleeds in acute ischemic stroke patients with atrial fibrillation and/or rheumatic heart disease. J Neurol Sci 369: 15-18.

69. Lasek-Bal A, Kowalewska-Twardela T, Gasior Z, Warsz-Wianecka A, Haberka M, et al. (2014) The significance of troponin elevation for the clinical course and outcome of first-ever ischaemic stroke. Cerebrovasc Dis 38(3): 212-218.

70. Hijazi Z, Oldgren J, Andersson U, Connolly SJ, Ezekowitz MD, et al. (2014) Importance of persistent elevation of cardiac biomarkers in atrial fibrillation: a RE-LY substudy. Heart 100(15): 1193-1200.

71. Scheitz JF, Erdur H, Haeusler KG, Audebert HJ2, Roser M, et al. (2015) Insular cortex lesions, cardiac troponin, and detection of previously unknown atrial fibrillation in acute ischemic stroke: insights from the troponin elevation in acute ischemic stroke study. Stroke 46(5): 1196-1201.
72. Ammann P, Maggiorini M, Bertel O, Haenseler E, Joller-Jemelka HI, et al. (2003) Troponin as a risk factor for mortality in critically ill patients without acute coronary syndromes. J Am Coll Cardiol 41(11): 2004-2009.

73. Bakshi TK, Choo MK, Edwards CC, Scott AG, Hart HH, et al. (2002) Causes of elevated troponin I with a normal coronary angiogram. Intern Med J 32(11): 520-525.

74. Wright RS, Williams BA, Cramner H, Gallahue F, Willmore T, et al. (2002) Elevations of cardiac troponin I are associated with increased short-term mortality in noncardiac critically ill emergency department patients. Am J Cardiol 90(6): 634-636.

75. Zellweger MJ, Schaer BA, Cron TA, Pfisterer ME, Osswald S (2003) Elevated troponin levels in absence of coronary artery disease after supraventricular tachycardia. Swiss Med Wkly 133(31-32): 439-441. 